\title{
Clinical Value of Emerging Bioanalytical Methods for Drug Measurements: A Scoping Review of Their Applicability for Medication Adherence and Therapeutic Drug Monitoring
}

\author{
Tanja R. Zijp ${ }^{1}$ (1) - Zamrotul Izzah ${ }^{1,2,3} \cdot$ Christoffer Åberg $^{3} \cdot$ C. Tji Gan ${ }^{4}$ Stephan J. L. Bakker • Daan J. Touw 1,3,6 . \\ Job F. M. van Boven ${ }^{1,6}$
}

Accepted: 26 September 2021 / Published online: 1 November 2021

(c) The Author(s) 2021

\begin{abstract}
Introduction Direct quantification of drug concentrations allows for medication adherence monitoring (MAM) and therapeutic drug monitoring (TDM). Multiple less invasive methods have been developed in recent years: dried blood spots (DBS), saliva, and hair analyses.

Aim To provide an overview of emerging drug quantification methods for MAM and TDM, focusing on the clinical validation of methods in patients prescribed chronic drug therapies.

Methods A scoping review was performed using a systematic search in three electronic databases covering the period 2000-2020. Screening and inclusion were performed by two independent reviewers in Rayyan. Data from the articles were aggregated in a REDCap database. The main outcome was clinical validity of methods based on study sample size, means of cross-validation, and method description. Outcomes were reported by matrix, therapeutic area and application (MAM and/or TDM).

Results A total of 4590 studies were identified and 175 articles were finally included; 57 on DBS, 66 on saliva and 55 on hair analyses. Most reports were in the fields of neurological diseases (37\%), infectious diseases (31\%), and transplantation $(14 \%)$. An overview of clinical validation was generated of all measured drugs. A total of 62 drugs assays were applied for MAM and 131 for TDM.

Conclusion MAM and TDM are increasingly possible without traditional invasive blood sampling: the strengths and limitations of DBS, saliva, and hair differ, but all have potential for valid and more convenient drug monitoring. To strengthen the quality and comparability of future evidence, standardisation of the clinical validation of the methods is recommended.
\end{abstract}

\section{Introduction}

Medication adherence is crucial for achieving optimal treatment effects [1,2]. Notably, failure to assess adherence may lead to under-treatment, potentially dangerous complications, and unnecessary complex or expensive second-line therapies [1, 2]. Medication adherence monitoring (MAM) is the periodical or routine assessment of patients'

Tanja R. Zijp and Zamrotul Izzah have contributed equally to this work and share first authorship.

Daan J. Touw and Job F. M. van Boven have contributed equally to this work and share last authorship.

Daan J. Touw

d.j.touw@umcg.nl

Extended author information available on the last page of the article medication-intake behaviour. Data on medication adherence allow clinicians to underpin their follow-up interventions and guide initiation of potential additional drugs. Currently, most clinicians rely on indirect measures, such as patient self-report, pill counts, and pharmacy-dispensing records, although it is widely recognised that these are subjective, non-granular, and prone to white-coat adherence, and therefore often overestimate true adherence [3, 4]. A more granular, but still indirect method to monitor medication intake information is MAM with electronic monitoring devices [5]. However, the most objective method to confirm adherence is to detect the actual presence of the drug in biological fluids.

Furthermore, an important stage of pharmacological patient management is the follow-up and optimisation of initial drug dosing. For a wide range of drugs, this can be done by monitoring drug concentrations [6]. Therapeutic drug monitoring (TDM) is the quantitative measurement of 


\section{Key Points}

This scoping review shows that dried blood spot, saliva, and hair are feasible for medication adherence and therapeutic drug monitoring in a wide range of chronically administered drugs and may improve the acceptability and accessibility of patient sampling. It recommends further research particularly in the fields of neurological diseases, respiratory diseases, transplantation and haemato-oncology.

A comprehensive overview is generated of drug assays with their current clinical validation status as appreciated by use of clinical samples, methods for validation, and the assessment for usability as stated in the publication.

Guidelines for clinical validation of emerging methods need to be developed further to improve the interpretation of their value, acceptability and use in routine care.

drug concentrations to assess adequate exposure, resistance, or side effects to medication [7]. This method is usually applied in conjunction with drugs that have a small therapeutic range, those that are known to exhibit high interindividual variation, and where a relationship between drug concentration and drug effect has been established. The main goal of TDM is dose optimisation, but it can also be used for the assessment of drug-drug interactions and medication adherence, where drug concentrations outside predefined ranges may indicate non-adherence [7].

Historically, MAM and TDM relied on classic venous blood sampling. However, venepuncture is burdensome for patients as it requires a visit to a clinic, needs to be performed by a phlebotomist or other healthcare professional, and is invasive, with associated pain and hematomas at the puncture site [8]. In some patient groups it is hard to sample blood, as veins may be hard to access or blood supply may be limited. For these patient groups and for general convenience, multiple emerging less invasive and non-invasive pre-analytical and bioanalytical methods have been developed. These upcoming methods include dried blood spots (DBS), urine, saliva, and hair analysis. A recent review has been published on the development of bioanalytical methods to measure drug concentrations in urine [9], leading to clear clinical consensus recommendations for urine testing of MAM or TDM $[10,11]$. Therefore, this review will focus on DBS, saliva, and hair as interesting matrices for drug monitoring.

Before any bioanalytical method can be implemented into clinical practice, the method needs to be analytically and clinically validated. In an analytical validation at least the linearity, accuracy and precision, matrix effect and stability of the method should be taken into account $[12,13]$. This should be followed by a clinical validation, where the concentrations measured in the alternative matrix samples are compared to other established methods (mostly blood or plasma concentrations), that are taken at the same time point, as recommended by the International Association for TDM and Clinical Toxicology (IATDMCT) for DBS validation [14]. While there are clear validation criteria for the analytical part, questions have been raised about whether the clinical validation receives sufficient attention and how it should be executed to verify its clinical usability [14].

For multiple chronic drug therapies (e.g., to treat human immunodeficiency virus (HIV), tuberculosis, epilepsy, etc.) the evidence for new measurement methods is growing and a translation from research to practice is expected to improve clinical outcomes in the near future. Especially during the global COVID-19 pandemic, an increased interest in remote (home) monitoring demands convenient methods for patients to provide samples themselves [15]. Between different therapeutic drug groups, however, there seem to be profound differences between validity and feasibility of applying these emerging drug-monitoring methods [6].

This scoping review provides an overview on the state-ofthe-art and clinical value of emerging non-invasive bioanalytical methods for drug-level monitoring. In particular, we focus on the use of these methods in real-life patient samples and whether they could be routinely applied to MAM and TDM of chronic drug therapies. We aim to give a high-level overview of the evidence in these fields and provide future research needs.

\section{Materials and Methods}

\subsection{Study Design}

A scoping review was performed to elucidate which chronically administered drugs are measured in DBS, saliva, and hair, the clinical validity of these methods, and their relevance for clinical decision making. A scoping review is an appropriate approach to explore evidence and knowledge gaps with the purposes defined by Arksey and O'Malley, namely to "examine the extent, range, and nature of research activity, to determine the value for undertaking a fully focused systematic review, to summarize and disseminate research findings, and to identify research gaps in the existing literature" [16]. Reporting was carried out in accordance with the Preferred Reporting Items for Systematic Reviews and Meta-Analysis extension for Scoping Reviews (PRISMA-ScR) [17] (Online Supplementary Information (OSM) 1). The protocol was registered before completion of the screening in the Open Science Platform under 
the registration https://doi.org/10.17605/OSF.IO/QJD9R (https://osf.io/qjd9r).

\subsection{Data Sources and Search Strategy}

The search was performed on 12 February 2020 in three electronic databases: Pubmed, Embase and Web of Science. The actual search terms used were '(non-)adherence', '(non-)compliance', 'drug monitoring', 'dried blood spots', 'urine', 'saliva', 'hair', 'concentration', 'assay', 'measurement', 'level', 'analysis', and 'quantification'. The only search filter applied was year of publication (after 2000). A follow-up search was performed on 10 March 2021 to include all articles up to 31 December 2020. The exact search queries are provided in OSM 2.

\subsection{Eligibility Criteria, Screening and Snowball Search}

All results from the search strategies were imported into the screening tool Rayyan [18], where the citations were checked for duplicates. All articles were screened for relevance based on title and abstract. This was followed by a review of the full-text articles. The inclusion and exclusion criteria were applied to the abstract and full-text articles. The screening and full-article eligibility assessment was performed by two independent reviewers (TRZ and ZI). In case of disagreement on inclusion, the article was discussed and could be assessed by a third independent reviewer (JFMvB) until consensus was reached. This study focuses on emerging methods, i.e. DBS, saliva, and hair monitoring, but excluding urine since urine was addressed in a previous review [9].

Articles that followed all of the following criteria were included: (1) the article used clinically applicable analytical methods to quantify drug compounds in patients' fingerprick DBS, saliva, and hair; (2) concentration levels were measured of medication that was prescribed for a duration of a minimum of 3 months and/or to treat a chronic disease; (3) the article was in English; and (4) the article was published between 2000 and 2020.

Articles were excluded when any of the following criteria were applicable: (1) reviews, protocols, case reports, no full-text available, (2) articles with a focus on toxicology, drug abuse, or unlicensed drugs, short-term, or single-dose drugs, (3) studies in healthy volunteers only, (4) studies that only analysed (clinical) samples or did not investigate the clinical applicability of the method in patients (e.g., TDM, precision medicine, adherence studies, cross-validation with a clinically used method), and (5) studies that reported fewer than ten patients using the drug of interest, which were considered case reports.

A snowball search was performed, where the following protocol was followed. A list was generated of all included studies with study ID, publication year, and matrix studied. Subsequently, from the articles published in the last 5 years (2016-2020), five articles per study matrix were randomly selected with the use of a true random-number generator (https://www.random.org/). Thereafter the reference lists in the randomly selected studies [19-33] were assessed to identify new articles of interest. Articles not identified with the previous searches that fulfilled the inclusion and not the exclusion criteria, were added to the Rayyan database for further analysis.

\subsection{Data Extraction}

All included articles were filed in a standardised form in a Research Electronic Data Capture (REDCap) database [34, 35], hosted by the University Medical Center Groningen (UMCG). The following study characteristics were extracted: (1) general article information (first author, title, publication year, continent); (2) assay methods (matrix, analytical method); (3) study population (disease group, subject description, patient population size), drugs measured (number of drugs tested, generic names of all drugs tested, number of patients that used the drug, metabolites tested), which was limited to drugs that have been used by ten or more patients; (4) assay information per matrix (validation characteristics, sample size per drug); and (5) clinical application (used for MAM and/or TDM, study outcomes to which the assay was linked). Data extraction was performed by one person and checked by a second person.

\subsubsection{Available Clinically Valid Methods for Chronically Used Drugs}

Before a drug assay can be clinically applied, the bioanalytical method must be analytically validated on the basis of guidelines provided by the European Medicines Agency (EMA) and/or the US Food and Drug Administration (FDA) $[12,13]$. Some clinical or intervention trials may have previously validated their assay method and described this analytical validation in a separate article. As it was outside of the scope of this review to assess the analytical details, these articles were not included. However, the existence of an analytical validation paper was registered in the RedCap database when its citation was available in the included article.

This review only included articles that described a clinical validation or that are reported to be used in clinical practice or within clinical trials, hence clinically valid. However, only a few guidelines or quality standards exist that describe how methods should be clinically validated, where the clinical validation of DBS has been described most [14]. At present, no guidelines exist for the clinical validation of saliva and hair. 


\subsubsection{Quality Assessment of Clinical Validation}

We developed quality criteria to classify all included studies on the basis of their clinical validity. The following factors were assessed to grade the quality of clinical validity: (1) sample size, (2) study methods for cross-validation, and (3) the opinion from the publishing authors on the clinical usability of their own developed method. For the sample size, the statistical value for patient and sample numbers have been described by Capiau et al., on behalf of IATDMCT [14], regarding DBS (40 patient samples or $\geq 25$ patients with multiple samples). The same sample-size criteria were adapted for saliva and hair. When either the number of samples or the number of patients was not available in the article, the assumptions were made that each patient provided one sample, or that each sample was provided by a unique patient.

The second criterion regarding study methods was specific for each matrix. For DBS and saliva, a cross-validation should be performed where the method is correlated with a previously validated analytical method in blood, plasma or serum, with bias assessed. For hair, a cross-validation with paired samples is less feasible, as body fluid levels change in time, while hair captures long-term exposure. Thus, validating hair studies could also be based on correlation with clinical parameters, such as dosage intake, average exposure over a certain time, or disease outcomes.

The last quality criterion was the opinion as expressed by the original authors of the included studies, which was assessed by any statements made in the article on the applicability of the method for clinical use. This part of the quality criteria was included because while some methods may have undergone full clinical validation, the results of this validation can be unconvincing or the authors can even conclude that the method is not feasible. These statements from the article were cited in the database, and graded as 'sure, applicable for clinical use', 'sure, not applicable for clinical use', and 'not sure (maybe or possibly) or no statements made'.

Each criterion was scored and the quality of clinical validation assays was finally labelled with different colours as described in the decision tree (Fig. 1). The colours represent the sum of criteria scores and show the degree of validity and clinical applicability. Note that the 'black' label refers to the authors' conclusions that the assay is not applicable in MAM and/or TDM, regardless of sample size or cross-over validation performed. Studies with the 'blue' label have the highest clinical validation quality for application in MAM and/or TDM followed by 'green' for valid application. A 'yellow' label is graded moderately valid, and 'red' less valid, both having a possible use for application in clinical settings.

\subsection{Outcomes}

We qualitatively evaluated the clinical applicability of noninvasive methods (DBS, saliva, and hair) either for MAM or TDM of chronically administered drugs. Furthermore, tables and graphs were created to reflect the characteristics of the included studies, including an overview of the drugs that were measured in the studies and their clinical validation status. Moreover, a figure was created to highlight the gaps in literature per bioanalytical method.

\subsection{Data Analysis}

Before data analysis, data quality was checked for discrepancies in the RedCap module. For the analysis, all data were downloaded from the RedCap and summarised in MS Excel for review and visual inspection. Data were grouped per study and per drug in R (RStudio version 1.4.1106). Descriptive statistics with absolute numbers per categories and percentages were used to describe the data.

\section{Results}

\subsection{Search and Selection of Studies}

Covering the period between 1 January 2000 and 31 December 2020, a total of 4590 studies were identified in the database searches on two separate occasions (Fig. 2). In the screening, records were removed on the basis of title and abstract; there were many records on bacterial adherence, biomarker quantification and reports on methods that only mentioned analytical validation in the abstract. After application of the exclusion criteria, articles on urine were removed, together with conference abstracts, case studies, studies where no real patient data were measured, and studies on drugs and patient populations that fell outside the scope of this review. A total of 175 articles were further analysed including four additional articles identified from the snowballing process. An overview of all included studies with citations is presented in OSM 3.

\subsection{Description of the Studies}

The general characteristics of all included studies are summarised in Table 1. Most studies reported on the measurement of a single drug in the paper that was considered for clinical validation $(n=121,69.1 \%)$, while some articles reported on simultaneously measured multi-drug methods. For both single- and multi-drug methods, only the drugs that were measured in ten or more patient samples were considered for this review, so the true number of drug assays mentioned in the publication may be higher in some studies [36, 


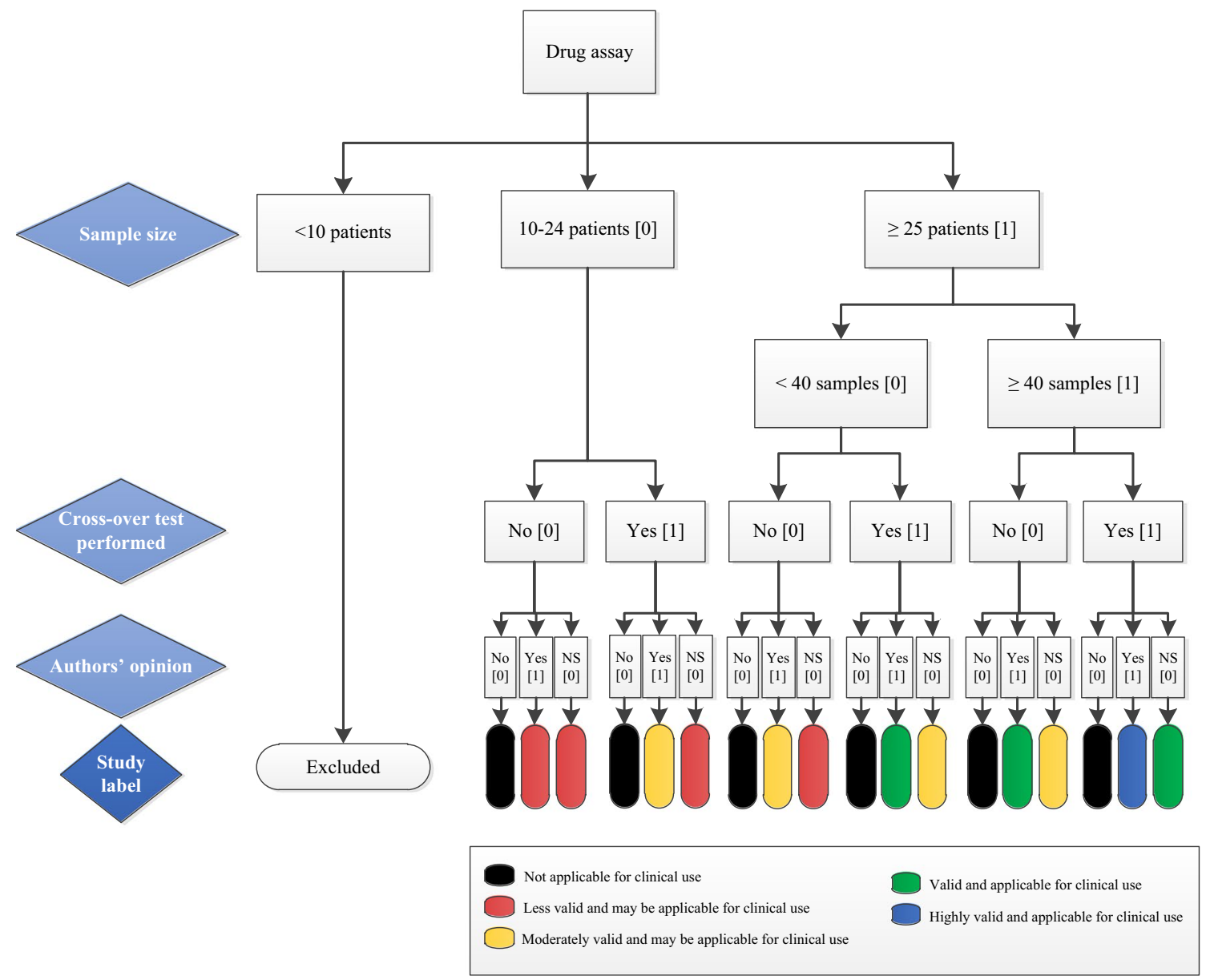

Fig. 1 Decision tree for labelling clinical validation quality of the bioanalytical assays. The study label is based on the sum scores of all quality criteria (scores of: $4=$ blue, $3=$ green, $2=$ yellow, and $0-1=$ red), except the black label, which refers to the conclusion of the authors of the included studies ("author's opinion") that the assay is not applicable for MAM and/or TDM regardless of other criteria.

37]. The median number of patients included in the studies was 43 and ranged from the minimum of ten to as many as 1165. Most studies were performed in adults and elderly patients, with $37(21 \%)$ articles that included neonates or children, and four (2\%) that specifically included pregnant or breastfeeding women. In some studies, multiple samples from the same patients were measured [19, 38-41].

The four most prominent clinical areas from which most articles were retrieved were neurological diseases, infectious diseases, transplantation medicine, and haemato-oncology. The emerging methods were majorly applied in neurological diseases, where epilepsy and psychiatric disorders were the most eminent diagnoses. Distinctions within the infectious disease groups were made in the diagnoses of HIV medication and tuberculosis, covering all but one included studies
Each study label therefore represents the degree of method validity and clinical applicability. The "author's opinion" refers to the original authors of the included studies. MAM medication adherence monitoring, $N S$ not sure (maybe, possibly) or no statements made, TDM therapeutic drug monitoring

on infection diseases (where the remaining study was on a doxycycline assay in hair) [42].

The numbers of included articles were evenly distributed between DBS $(n=57)$, saliva $(n=66)$ and hair $(n=$ 67). Most articles reported on the measurement of drugs in only one matrix of interest. Some articles combined several matrices of interest [43-45], or included other experimental methods such as sweat [46, 47], nasal mucus [48], volumetric absorptive microsampling (VAMS) [49], finger smears, and scalp swabs [31]. The majority of the articles ( $n$ $=129,73.7 \%$ ) measured the drugs in simultaneously taken venepuncture-acquired blood samples to compare the new method to an established method.

The ultimate clinical applicability for the described methods, either for MAM or for TDM, was assessed for each article. Most articles on DBS were performed with a focus 


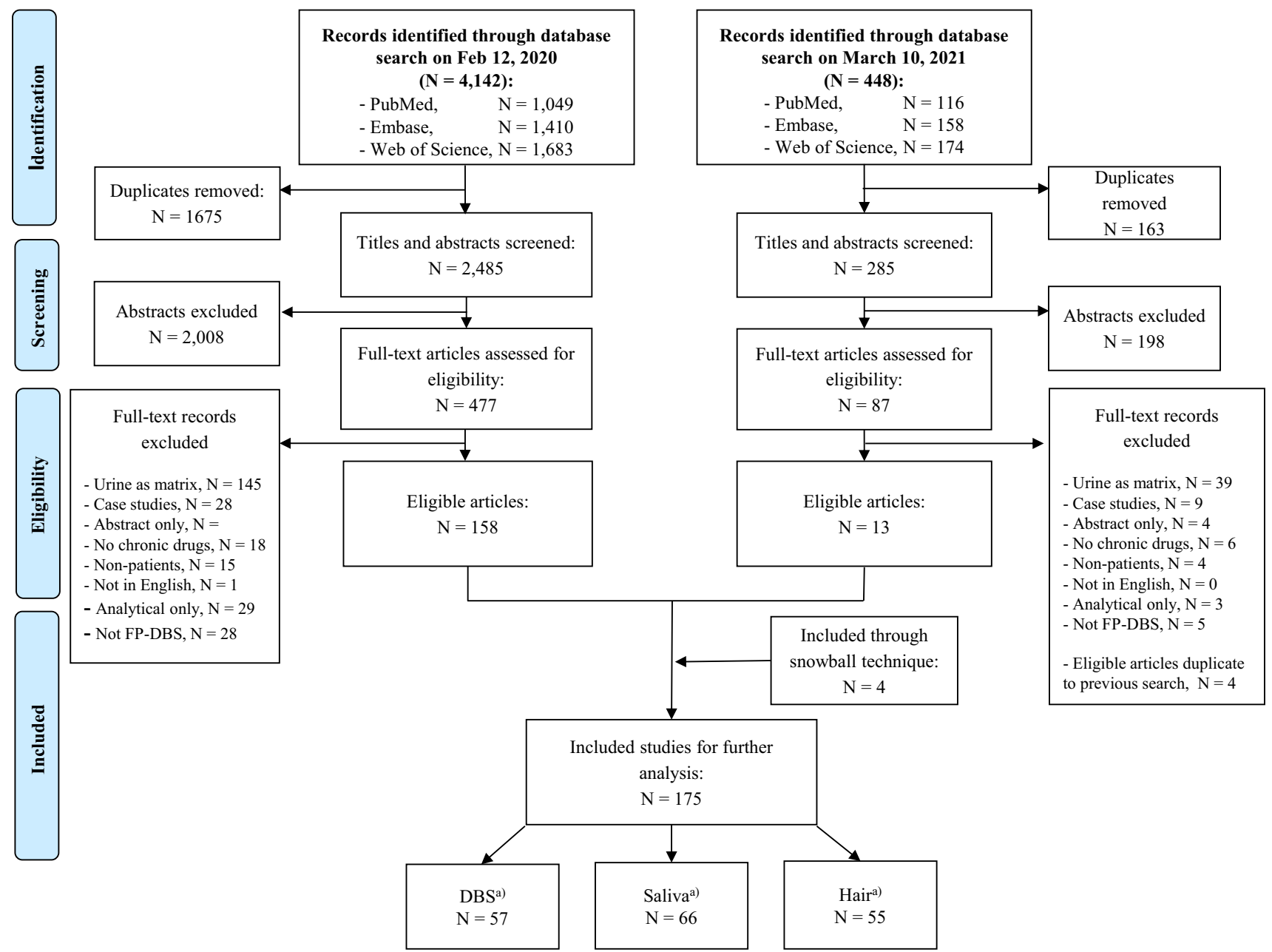

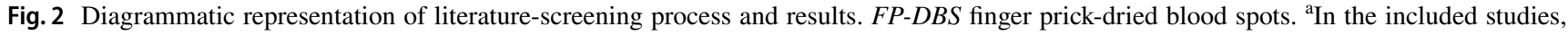
one article described both hair and DBS, two articles described saliva and hair analysis simultaneously

on TDM ( $n=52,91 \%$, of which three also showed possible application for MAM). Similarly, for saliva the majority $(n=47,71 \%)$ focused on an applicability for TDM or for both MAM and TDM $(n=10,15 \%)$. However, for hair, the most research was performed on long-term adherence and therefore applicability for MAM $(n=35,64 \%)$. Only 17 (31\%) articles researched whether hair could predict clinical outcomes and therefore be suitable for TDM, where seven articles (13\%) had shared focus on MAM and TDM.

The literature on clinically validated assay methods in DBS, saliva, and hair has considerably increased in the last few years. For example, half of the included articles were published in the last 5 years (2016-2020), and approximately $80 \%$ in the last decade (2011-2020). In geographical terms, half of the articles (50\%) were published in Europe. Asia and North America delivered comparable numbers of publications, with $16 \%$ and $14 \%$ of the included articles, respectively.
The applicability for which each method was proposed in MAM or TDM, for each publication year, and the number of articles in the major specific therapeutic groups are shown in Figs. 3 and 4, respectively.

\subsection{Clinical Validation Quality of the Drug Assays}

A total of 219 drug assays described in the articles were evaluated for clinical validity (Table 2), with the quality criteria as described previously and classification of the colours in accordance with Fig. 1. Assays with under ten patients or patient samples were excluded. A total of 121 assays were performed with samples from under 25 patients. For 139 assays, samples from 25 or more patients were used, of which 100 assays $(63 \%)$ were validated with more than 40 patient samples. For 31 assays, either the number of patients or the number of samples was not available.

Cross-validation was rated as the assessment of a correlation with either a previously validated assay method or 
Table 1 Characteristics of the included studies $(N=175)$

\begin{tabular}{|c|c|}
\hline Parameter & $N(\%)$ \\
\hline Number of drugs clinically validated, median (range) & $1(1-9)$ \\
\hline Sample size, median (range) & $43(10-1165)$ \\
\hline \multicolumn{2}{|l|}{ Study population } \\
\hline Adults and elderly & $134(76.6)$ \\
\hline Paediatrics & $37(21.1)$ \\
\hline Pregnant or breastfeeding women & $4(2.3)$ \\
\hline \multicolumn{2}{|l|}{ Most reported fields } \\
\hline Neurological diseases & $64(36.6)$ \\
\hline Epilepsy & $27(15.4)$ \\
\hline Pain & $6(3.4)$ \\
\hline Parkinson's disease & $1(0.6)$ \\
\hline Psychiatry & $30(17.1)$ \\
\hline Infectious diseases & $54(30.8)$ \\
\hline HIV/AIDS & $44(25.1)$ \\
\hline Tuberculosis & $10(5.7)$ \\
\hline Transplantation & $24(13.7)$ \\
\hline (Haemato-)Oncology & $14(8.0)$ \\
\hline Other $^{\mathrm{a}}$ & $19(10.8)$ \\
\hline \multicolumn{2}{|l|}{ Matrix } \\
\hline DBS & $57(32.5)$ \\
\hline Saliva & $66(37.7)$ \\
\hline Hair & $55(31.4)$ \\
\hline \multicolumn{2}{|l|}{ Proposed applicability of method ${ }^{\mathrm{b}}$} \\
\hline MAM & $62(35.4)$ \\
\hline TDM & $131(74.9)$ \\
\hline \multicolumn{2}{|l|}{ Publication year } \\
\hline $2000-2005$ & $18(10.3)$ \\
\hline 2006-2010 & $18(10.3)$ \\
\hline $2011-2015$ & $51(29.1)$ \\
\hline 2016-2020 & $88(50.3)$ \\
\hline \multicolumn{2}{|l|}{ Geographical area } \\
\hline Europe & $88(50.3)$ \\
\hline Asia & $28(16.0)$ \\
\hline North America & $25(14.3)$ \\
\hline Africa & $17(9.7)$ \\
\hline South America & $14(8.0)$ \\
\hline Oceania & $3(1.7)$ \\
\hline
\end{tabular}

AIDS acquired immunodeficiency syndrome, $D B S$ dried blood spots, $H I V$ human immunodeficiency virus, MAM medication adherence monitoring, TDM therapeutic drug monitoring

${ }^{a}$ Other: cardiovascular disease $(7,4.0 \%)$, inflammatory bowel disease $(3,1.7 \%)$, asthma/chronic obstructive pulmonary disease $(2,1.1 \%)$, chronic migraine $(2,1.1 \%)$, systemic inflammatory disease $(2,1.1 \%)$, chronic hyposmia $(1,0.6 \%)$, diabetes mellitus $(1,0.6 \%)$, Q fever or Whipple's disease $(1,0.6 \%)$

bighteen studies described the applicability of the method in both TDM and MAM a clinical or adherence outcome. Of the drug assays, $48 \mathrm{did}$ not describe a full cross-validation, which was a result of the article not reporting on the linearity of the method, or not describing a bias assessment with a Bland Altman plot (for 24 DBS articles). For DBS, only five articles were without venous measurement as comparator [50-53]. These articles described a new detection method [50], compared the results with adherence outcomes (self-report, prescription refills, pill counts, or combinations) [51, 52], or applied DBS in a clinically usable pharmacokinetic model for TDM.

In the opinion of the authors of the studies with regard to the methods, most assays were stated to be suitable for at least one of the clinical applications mentioned $(n=185$, $84.5 \%)$. For 25 assays (11.4\%), the authors concluded that the method was not clinically applicable. For some methods, the authors were uncertain about the clinical applicability of the assay $(n=60,27 \%)$, or did not describe any conclusion on usability $(n=21,9.6 \%)$.

\subsection{Drug Measurements}

A total of 118 unique drugs that were measured in DBS, saliva, and hair were considered in this review. A list of these drugs was ordered by the Anatomical Therapeutic Chemical (ATC) classification system and grouped by the ATC level that was considered appropriate for displaying the results. An overview of all drugs measured, including the number of assays that assessed the drug and the highest clinical validation status achieved for MAM and TDM, is presented in Table 2. For drugs with several studies on the same clinical application and matrix, the colour of the dot represents the colour of the highest clinical validation status.

Most non-invasive methods were valid and (possibly) applicable for MAM in clinical settings. No reported assays were infeasible for MAM. Furthermore, highly valid methods were available for 16 drugs measured for MAM mostly in epilepsy, HIV/AIDS, and pain [21, 52, 54-65]. Hair is the most widely used method to detect the presence of 37 unique drugs and/or their metabolites, followed by saliva (31 drugs) and DBS (20 drugs). The emerging matrices may also be applied to detect intake adherence and changes in drugtaking behaviour, such as mentioned for aripiprazole and pipamperone in DBS [66], amisulpride [67], aripiprazole and olanzapine [68] in saliva, and ciclosporin in hair [69].

On the other hand, the applicability of less invasive methods for TDM in clinical practice varied. There is evidence that TDM of the following drugs is not feasible using DBS (aripiprazole, pipamperone, risperidone) [66], saliva (amisulpride, aripiprazole, lisdexamfetamine, moxifloxacin, 


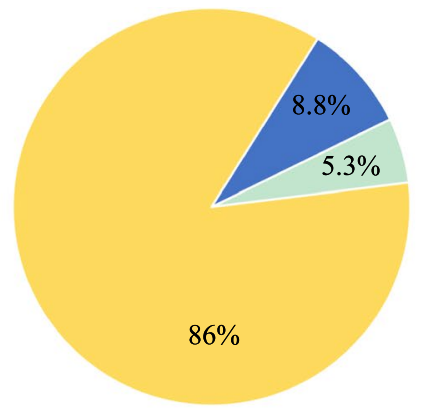

DBS

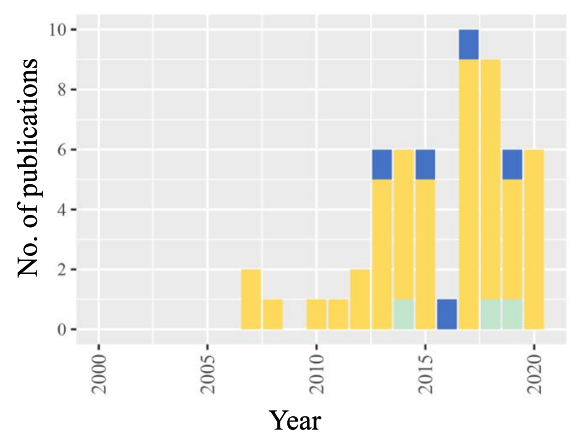

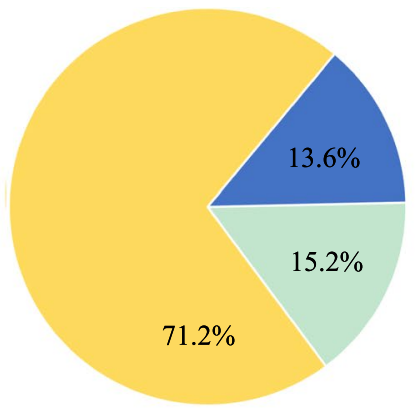

Saliva

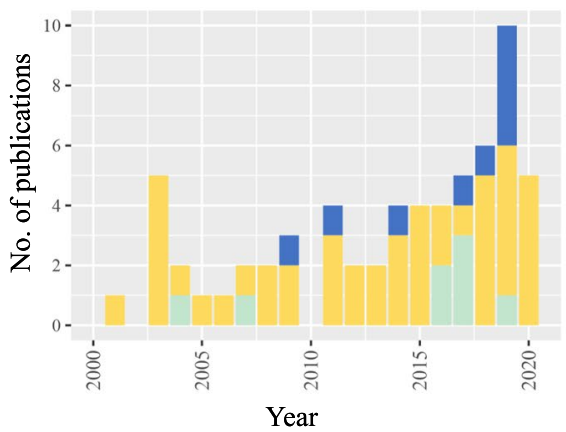

Clinical applicability

MAM

TDM

MAM and TDM

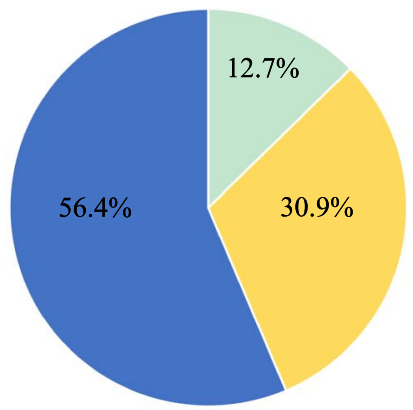

Hair

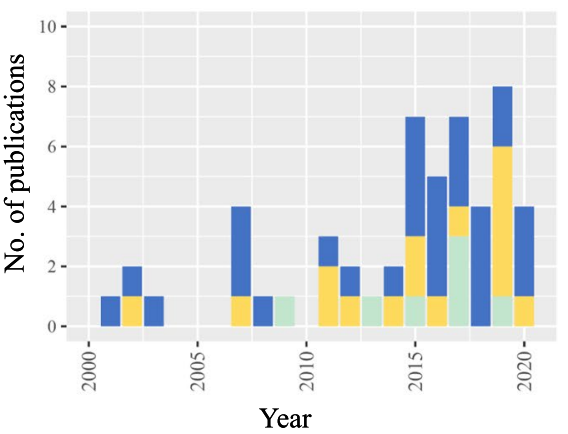

Fig. 3 Emerging matrices with proposed applicability of the method. The top figures show the application of each matrix for MAM, TDM or both MAM and TDM, while the bottom figures display the dis- tribution of each matrix application per publication year. $D B S$ dried blood spots, MAM medication adherence monitoring, TDM therapeutic drug monitoring
Fig. 4 Bubble plot depicting knowledge gaps. Bubble size represents the number of published articles for each analysis method and the plot is ordered by therapeutic area. Other disease group refers to: cardiovascular diseases, inflammatory bowel diseases, asthma/ chronic obstructive pulmonary disease, chronic migraine, systemic inflammatory disease, chronic hyposmia, diabetes mellitus, and Q fever or Whipple's disease. $D B S$ dried blood spots

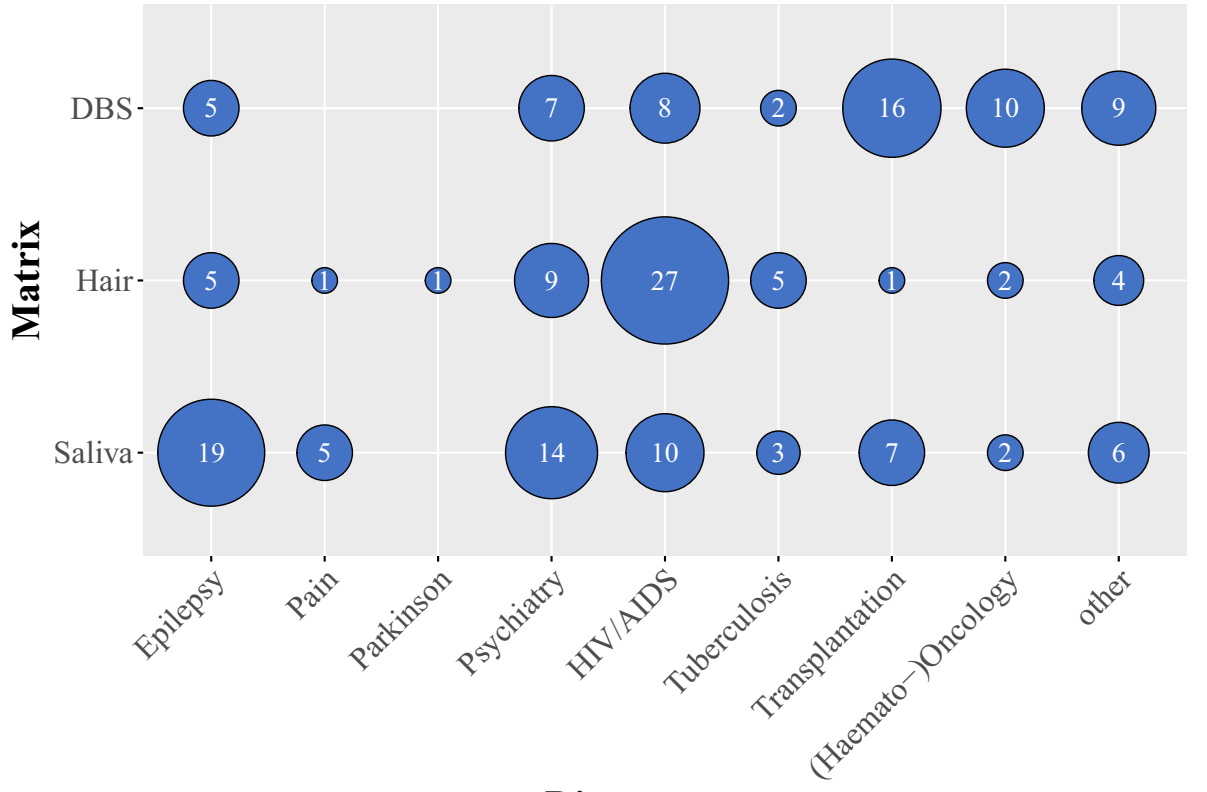

Disease group 
Table 2 Drugs with their highest assessed clinical validation status in dried bloods spots (DBS), saliva, and hair and whether these methods have been used for medication adherence monitoring (MAM) or therapeutic drug monitoring (TDM)

\begin{tabular}{|c|c|c|c|c|c|c|c|}
\hline \multirow{2}{*}{ Drug } & \multirow{2}{*}{$\begin{array}{c}\text { No. of } \\
\text { assays }^{\text {a }}\end{array}$} & \multicolumn{2}{|c|}{ DBS } & \multicolumn{2}{|c|}{ Saliva } & \multicolumn{2}{|c|}{ Hair } \\
\hline & & MAM & TDM & MAM & TDM & MAM & TDM \\
\hline \multicolumn{8}{|c|}{ Blood glucose lowering drugs } \\
\hline metformin & 1 & & & & & & \\
\hline sitagliptin & 1 & & & & & & \\
\hline \multicolumn{8}{|c|}{ Antithrombotic agents } \\
\hline rivaroxaban & 1 & & & & & & \\
\hline warfarin & 2 & & & & & & \\
\hline \multicolumn{8}{|c|}{ Cardiovascular system } \\
\hline ambrisentan & 1 & & & & & & \\
\hline amlodipine & 2 & & & & & & \\
\hline bisoprolol & 1 & & & & & & \\
\hline bosentan & 1 & & & & & & \\
\hline digoxin & 1 & & & & & & \\
\hline enalapril & 1 & & & & & & \\
\hline hydrochlorothiazide & 2 & & & & & & \\
\hline losartan & 1 & & & & & & \\
\hline metoprolol & 1 & & & & & & \\
\hline moxonidine & 1 & & & & & & \\
\hline nifedipine & 1 & & & & & & \\
\hline perindopril & 1 & & & & & & \\
\hline ramipril & 1 & & & & & & \\
\hline sildenafil & 1 & & & & & & \\
\hline spironolactone & 1 & & & & & & \\
\hline tadalafil & 1 & & & & & & \\
\hline torasemide & 1 & & & & & & \\
\hline valsartan & 2 & & & & & & \\
\hline \multicolumn{8}{|l|}{ Glucocorticoids } \\
\hline prednisolone & 1 & & & & & & \\
\hline \multicolumn{8}{|c|}{ Antibacterials for systemic use } \\
\hline doxycycline & 1 & & & & & & \\
\hline levofloxacin & 2 & & & & & & \\
\hline linezolid & 1 & & & & & & \\
\hline moxifloxacin & 2 & & & & & & \\
\hline \multicolumn{8}{|l|}{ Antimycobacterials } \\
\hline bedaquiline & 1 & & & & & & \\
\hline clofazimine & 1 & & & & & & \\
\hline ethambutol & 1 & & & & & & \\
\hline ethionamide & 1 & & & & & & \\
\hline isoniazid & 5 & & & & & & \\
\hline pyrazinamide & 2 & & & & & & \\
\hline rifampicin & 3 & & 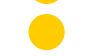 & & 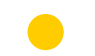 & & \\
\hline
\end{tabular}


Table 2 (continued)

\begin{tabular}{|c|c|c|c|c|c|c|c|}
\hline \multirow{2}{*}{ Drug } & \multirow{2}{*}{$\begin{array}{c}\text { No. of } \\
\text { assays }^{\mathrm{a}}\end{array}$} & \multicolumn{2}{|c|}{ DBS } & \multicolumn{2}{|c|}{ Saliva } & \multicolumn{2}{|c|}{ Hair } \\
\hline & & MAM & TDM & MAM & TDM & MAM & TDM \\
\hline \multicolumn{8}{|c|}{ Antivirals for systemic use } \\
\hline abacavir & 1 & & & & & & \\
\hline atazanavir & 6 & & & & & & \\
\hline darunavir & 1 & & & & & & \\
\hline efavirenz & 11 & & & & & & \\
\hline emtricitabine & 4 & & & & & & \\
\hline indinavir & 4 & & & & & & \\
\hline lamivudine & 5 & & & & & & \\
\hline lopinavir & 6 & & & & & & \\
\hline nevirapine & 16 & & & & & & \\
\hline raltegravir & 1 & & & & & & \\
\hline ritonavir & 5 & & & & & & \\
\hline tenofovir & 10 & & & & & & \\
\hline zidovudine & 2 & & & & & & \\
\hline \multicolumn{8}{|c|}{ Antineoplastic agents } \\
\hline docetaxel & 1 & & & & & & \\
\hline epirubicin & 1 & & & & & & \\
\hline erlotinib & 1 & & & & & & \\
\hline gemcitabine & 1 & & & & & & \\
\hline imatinib & 2 & & & & & & \\
\hline mercaptopurine & 1 & & & & & & \\
\hline methotrexate & 1 & & & & & & \\
\hline nilotinib & 1 & & & & & & \\
\hline paclitaxel & 1 & & & & & & \\
\hline pazopanib & 1 & & & & & & \\
\hline radotinib & 1 & & & & & & \\
\hline topotecan & 1 & & & & & & \\
\hline \multicolumn{8}{|l|}{ Endocrine therapy } \\
\hline abiraterone & 1 & & & & & & \\
\hline tamoxifen & 2 & & & & & & \\
\hline \multicolumn{8}{|c|}{ Immunosuppressants } \\
\hline adalimumab & 1 & & & & & & \\
\hline azathioprine & 1 & & & & & & \\
\hline ciclosporin & 6 & & & & & & \\
\hline everolimus & 2 & & & & & & \\
\hline golimumab & 1 & & & & & & \\
\hline mycophenolic acid & 6 & & & & & & \\
\hline sirolimus & 2 & & & & & & \\
\hline tacrolimus & 14 & & & & & & \\
\hline vedolizumab & 1 & & & & & & \\
\hline \multicolumn{8}{|l|}{ Opioids } \\
\hline buprenorphine & 3 & & & & & & \\
\hline fentanyl & 2 & & & & & & \\
\hline methadone & 4 & & & & & & \\
\hline morphine & 2 & & & & & & \\
\hline oxycodone & 3 & & & & & & \\
\hline tramadol & 1 & & & & & & \\
\hline
\end{tabular}


Table 2 (continued)

\begin{tabular}{|c|c|c|c|c|c|c|c|}
\hline \multirow{2}{*}{ Drug } & \multirow{2}{*}{$\begin{array}{c}\text { No. of } \\
\text { assays }^{\text {a }}\end{array}$} & \multicolumn{2}{|c|}{ DBS } & \multicolumn{2}{|c|}{ Saliva } & \multicolumn{2}{|c|}{ Hair } \\
\hline & & MAM & TDM & MAM & TDM & MAM & TDM \\
\hline \multicolumn{8}{|c|}{ Antimigraine preparations } \\
\hline almotriptan & 1 & & & & & & \\
\hline eletriptan & 1 & & & & & & \\
\hline frovatriptan & 1 & & & & & & \\
\hline rizatriptan & 1 & & & & & & \\
\hline sumatriptan & 1 & & & & & & \\
\hline \multicolumn{8}{|l|}{ Antiepileptics } \\
\hline carbamazepine & 9 & & & & & & \\
\hline lacosamide & 2 & & & & & & \\
\hline lamotrigine & 7 & & & & & & \\
\hline levetiracetam & 6 & & & & & & \\
\hline oxcarbazepine & 1 & & & & & & \\
\hline perampanel & 1 & & & & & & \\
\hline phenobarbital & 1 & & & & & & \\
\hline phenytoin & 2 & & & & & & \\
\hline pregabalin & 1 & & & & & & \\
\hline rufinamide & 1 & & & & & & \\
\hline topiramate & 3 & & & & & & \\
\hline valproic acid & 7 & & & & & & \\
\hline \multicolumn{8}{|l|}{ Anti-parkinson } \\
\hline selegiline & 1 & & & & & & \\
\hline \multicolumn{8}{|c|}{ Antipsychotics and anxiolytics (psycholeptics) } \\
\hline amisulpride & 2 & & & & & & \\
\hline aripiprazole & 3 & & & & & & \\
\hline chlorpromazine & 1 & & & & & & \\
\hline cloxazolam & 1 & & & & & & \\
\hline clozapine & 6 & & & & & & \\
\hline haloperidol & 1 & & & & & & \\
\hline lithium & 1 & & & & & & \\
\hline olanzapine & 2 & & & & & & \\
\hline pipamperone & 2 & & & & & & \\
\hline quetiapine & 4 & & & & & & \\
\hline risperidone & 7 & & & & & & \\
\hline ziprasidone & 1 & & & & & & \\
\hline \multicolumn{8}{|l|}{ Antidepressants } \\
\hline amitriptyline & 1 & & & & & & \\
\hline citalopram & 4 & & & & & & \\
\hline duloxetine & 2 & & & & & & \\
\hline escitalopram & 1 & & & & & & \\
\hline fluoxetine & 1 & & & & & & \\
\hline mirtazapine & 2 & & & & & & \\
\hline venlafaxine & 3 & & & & & & \\
\hline \multicolumn{8}{|c|}{ Centrally acting sympathomimetics } \\
\hline lisdexamfetamine & 2 & & & & & & \\
\hline methylphenidate & 5 & & & & & & \\
\hline
\end{tabular}


Table 2 (continued)

\begin{tabular}{|c|c|c|c|c|c|c|c|}
\hline \multirow{2}{*}{ Drug } & \multirow{2}{*}{$\begin{array}{c}\text { No. of } \\
\text { assays }^{\text {a }}\end{array}$} & \multicolumn{2}{|c|}{ DBS } & \multicolumn{2}{|c|}{ Saliva } & \multicolumn{2}{|c|}{ Hair } \\
\hline & & MAM & TDM & MAM & TDM & MAM & TDM \\
\hline \multicolumn{8}{|c|}{ Obstructive airway diseases } \\
\hline $\begin{array}{l}\text { formoterol } \\
\text { theophylline }\end{array}$ & $\begin{array}{l}1 \\
2\end{array}$ & & & & & & \\
\hline
\end{tabular}

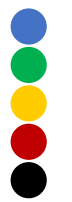

Highly valid and applicable for clinical use

Valid and applicable for clinical use

Moderately valid and may be applicable for clinical use

Less valid and may be applicable for clinical use

Not applicable for clinical use

${ }^{a}$ The number of assays refers to the total number of publications that describe this drug assay for the mentioned methods

${ }^{b}$ For drugs with several studies the dot represents the study with the highest clinical validation status, as determined according to Fig. 1

olanzapine, oxycodone) [37, 66-68, 70, 71], and hair (chlorpromazine, ciclosporin, erlotinib, lamotrigine) $[29,45,69$, 72]. These alternative matrices could not substitute traditional blood samples for TDM purposes due to large interindividual or intraindividual variability and poor correlations [37, 66-68, 71, 73-75].

\section{Discussion}

This review provides an overview of the clinical applicability of emerging bioanalytical methods and evidence that MAM or TDM using DBS, saliva, or hair is feasible for a wide range of chronically administered drugs. Using these alternative matrices may improve the acceptability and accessibility for patient sampling and reduce the burden and costs of drug monitoring in routine practice. Moreover, there is a shift from the experimental setting to clinical practice, and research on clinical application of these less invasive and potentially home-based methods is becoming more prominent. Notably, we found that the main focus for the use of DBS and saliva sampling was dose optimisation for TDM, in contrast to hair, which was mostly applied for long-term MAM. The findings highlight that most research is concentrated on saliva (in neurological diseases) followed by DBS (in transplantation and oncology settings) and hair (for HIV/AIDS).

\subsection{Clinical Application}

Drug measurements can be applied in two ways: to determine if the drug is present in the body (qualitative) and to determine what the concentration is (quantitative). For MAM, the most conventional approach to decide if the patient is adherent is using a qualitative approach, while for
TDM, by definition, a quantitative result is needed as the drug level will be assessed to find out if a treatment target is reached.

In most research, MAM is defined as either above or below a threshold value. The easiest way to define nonadherence would be a drug level under the lower limit of quantification (LLOQ) for the analytical method, where drug levels are undetectable and therefore considered not present. For this method, the sensitivity of the detection method should be sufficiently low, to also detect, for example, low trough levels in the case of a drug with a short half-life. For saliva and dried blood spots, the absence of the drug in the matrix would mean the drug was not taken for several halflives [51, 52, 67, 76, 77]. Therefore, white-coat adherence, which is the intake of medication just before a clinical visit, cannot be detected. For hair, the absence of the drug would mean the drug was not or was only very limitedly taken for longer periods [33, 57, 64, 65, 78].

More refined strategies to monitor adherence are with a quantitative approach by identifying a threshold, under which drug levels are sub-optimal, and the risk due to a patient being non-adherent is high $[33,51,52,57,63,65$, 77]. As all drug levels are dependent on the pharmacokinetics of the individual patients, where metabolizing-enhancing factors, such as pharmacogenetics (rapid metabolizers), drug-drug interactions (e.g., induction), and also causes for low absorption of the drug (e.g., sickness or diarrhoea) could lead to unexpectedly low concentrations [7, 53, 79-82]. Therefore, not all patients with low DBS, salivary or hair concentrations may be non-adherent when drug levels are below the defined threshold. For some drugs, the presence or absence of metabolites or the ratio between metabolite and parent drug could distinguish non-adherence from a fast metabolisation profile [83]. Furthermore, high drug levels could also be indicative of non-adherence, such as 
overdosing or taking a dose just before a clinical visit [81]. The times of both sampling and drug intake should be considered when interpreting the drug measurements.

For TDM, drug measurements enable tuning drug dosages to prevent toxicity and undertreatment for drugs with narrow therapeutic ranges. In this review, TDM is indicated in a number of drugs to enable safe and efficient use, such as immunosuppressants, antiepileptics, antifungals, antiretrovirals and antibiotics. There should be a good correlation between dose-dependent concentrations and clinical outcomes. Some articles described a validation for TDM that was not sufficient, as the relationship was poor due to, for example, large interindividual or intraindividual variability [37, 66-68, 71, 73-75], but still recommend uisng their validated assay method for (semi-) quantitative MAM. They strongly suggest applying the matrices as qualitative adherence tools in clinical practice, for example DBS for risperidone, saliva for lisdexamfetamine [70], and hair for lamotrigine [45].

The choice of which matrix to be used for MAM or TDM depends on the purpose of the measurements, the population (e.g., paediatrics, elderly), disease state, eventual logistic barriers (costs, apparatus, personnel, remote location, possible storage environment), drug characteristics (physical/ chemical properties, dosage form, stability), for which more details can be found in the next section. The choice of matrix for a specific drug needs a validated drug assay method and could be made by the clinician on basis of the results summarised in Table 2. Please note that when there are previous clinically validated methods available, they may not be directly transferrable to all patient groups and should be revalidated.

\subsection{Quality Assessment Per Matrix}

A novel quality-assessment tool for clinical validation of the assays was developed for this review to provide a quick overview of the clinical validity using three main points, namely sample size, performance of a cross-over test with a previously validated method, and the opinion of the authors that published the bioanalytical assay; the requirements for the first two are described in the IATDMCT DBS guideline [14]. The results indicated profound variation in the extent to which clinical validation was performed. However, most articles included in this study did have sufficiently high standards. Regarding sample size, most articles included sufficient patients and samples. The use of at least 40 patient samples is recommended for clinical validation to account for statistical variation and matrix effects [14]. Lower numbers may not be sufficient to detect bias and imprecision.

Most articles performed a clinical validation with (simultaneously drawn) blood samples where they applied correlation and bias assessment with weighted Deming or Passing
Bablok regression and Bland-Altman analysis. For a successful clinical validation, there should be good agreement between the emerging methods and either blood concentrations or clinical outcomes where acceptance limits of such analysis should be fulfilled. When an assay did not meet all pre-defined criteria for TDM, some authors concluded that it could still be used in clinical practice, for example as a medication adherence tool [45, 66-70]. If the assay can accurately detect and measure drug concentrations in the emerging matrices, it can show that the drug has been taken by the patient.

Consequently, many assay methods were found to be feasible for clinical application, as $85 \%$ of the article selfreported the assay method to be clinically feasible for use. Of all analyses included, most (76\%) were sufficiently suitable. Details on the methods, their suitability, and their specific feasibility for implementation are discussed further in each matrix below.

\subsubsection{Dried Blood Spots}

DBS can be obtained by finger prick or capillary blood spotted onto a filter card and dried at room temperature. DBS levels can be applied to predict drug full blood or plasma levels. Yet, they are not necessarily equal or proportional to blood levels because of binding of the drugs to blood components or differences in drug concentrations between venous and capillary blood [84]. DBS is particularly helpful for paediatric and elderly patients and patients who have difficulty travelling because this method requires small sample volumes $(10-30 \mu \mathrm{L})$ and can be performed at home. The collection of DBS samples has been applied in studies in remote or resource-limited settings, particularly in places where uninterrupted cold chains were not always available. However, bioanalytical issues such as lower sensitivity, analytical bias due to variability of haematocrit values, and the complexity of extraction and recovery may limit the acceptance of the DBS technique [28, 85].

In this review, DBS has been shown to be widely used in transplantation and haemato-oncology patients to monitor therapeutic ranges [27, 40, 86-92]. DBS is also suitable for TDM of tacrolimus in paediatric transplant recipients [93]. Home DBS could detect variability in tacrolimus concentrations in both pre-adolescent and adolescent transplant recipients over time [39]. Therefore, future studies may address cost evaluation and efficacy of DBS in clinical practice to investigate the possible impact of logistical errors and incorrect home DBS sampling by patients [27, 89, 94].

Most studies included in this review $(n=44,77 \%)$ discussed the haematocrit effect on measured drug concentrations, and some even used patient-specific haematocrit values for calculating theoretical blood levels [28, 92, 95-97]. Recently, alternative DBS methods have been introduced to 
increase the extraction efficiency and to overcome problems associated with DBS samples [98]. For instance, the volumetric absorptive microsampling (VAMS) approach overcomes the haematocrit effect, spot irregularity, and volume bias associated with finger prick DBS [49, 99]. However, some technical problems were addressed by Veenhof et al. [49], namely that the absence of anticoagulant in patient samples, VAMS batch differences, and the invisible undersampling of VAMS samples lowered the extraction recovery compared to DBS samplings. New sampling devices such as volumetric absorptive paper discs (VAPD) and mini-discs (VAPDmini) have been introduced using the combined innovation principles of DBS and VAMS [100].

\subsubsection{Saliva}

In recent years, there has been a growth in clinical interest in saliva as a matrix for bioanalysis [101]. Saliva is considered a patient-friendly alternative matrix to monitor drug exposure and adherence because of the ease of sampling and being less invasive. Saliva can be obtained by non-stimulated (direct collection into a tube) or stimulated (using a cotton swab with citric acid) methods. Penetration of drugs into saliva is predominantly driven by passive diffusion. Only free unionized and non-protein-bound drugs can penetrate into saliva. For instance, antiretroviral drugs that are not extensively bound to protein may penetrate well into saliva $[73,102]$. Therefore, salivary drug concentrations can be used for extrapolation to unbound plasma concentrations.

Apart from the degree of protein binding, other factors that can affect the passage of drugs from blood to saliva are salivary flow rate, salivary and blood $\mathrm{pH}$, as well as the ionization constant of an acidic drug (pKa), molecular weight, and lipid solubility of the analyte [101, 103]. The degree of ionization of a substance would determine if the saliva to plasma ratio remains unaffected by saliva $\mathrm{pH}$ (normally in the range of 6.2-7.4), for instance, saliva to plasma ratio of neutral drugs or those with a $\mathrm{p} K_{\mathrm{a}}$ below 5.5 or above 8.5 should not be affected by salivary $\mathrm{pH}$ variation [104]. However, if citric acid is used to stimulate oral fluid production at a faster rate, there might be a change in $\mathrm{pH}$ and the concentration of bicarbonate, which affects the drug concentration in a $\mathrm{pH}$-dependent manner [105]. The detection of drugs in saliva will also depend on other factors such as dose, frequency of exposure and limit of detection of analytical assays [101]. Clear reference values are needed to discriminate between therapeutic and subtherapeutic concentrations.

Saliva is widely chosen as a preferred matrix in patients with epilepsy, psychiatric disorders, and HIV/AIDS. Interestingly, collecting oral fluids from patients in the first two groups is difficult. These patients are rapidly agitated and may experience a dry mouth due to the anticholinergic side effects of, for example, clozapine, olanzapine, and risperidone [68]. Also, high variations have been observed in saliva versus serum concentrations [67, 68]. Large interindividual or intraindividual variations were affected by salivary $\mathrm{pH}$ or saliva being contaminated by drug residues in the mouth resulting in poor correlations with plasma or serum concentrations [67, 70]. A study by Mendonza [104] found that teeth brushing and flossing led to some degree of bleeding and therefore contamination of saliva with blood. This contamination resulted in high mycophenolic acid concentrations at morning trough sampling times. However, this study did not assess the possibility of overcoming this problem by rinsing the mouth. Another recent study by Ghareeb [106] revealed that reducing blood contamination in the saliva by rinsing the oral cavity with water before sampling could improve the correlation coefficient between tacrolimus concentrations in oral fluid and whole blood. Another problem has been observed in some antiretroviral and antihypertensive drugs that are detected in very low concentrations in saliva [107, 108]. For example, tenofovir is poorly diffused in saliva because it is almost completely ionized under physiological conditions and the ionized form has difficulty passing through the salivary glands [107]. Regarding the antihypertensives, enalapril, lisinopril, and ramipril could not be quantified due to the fact that these drugs are not excreted, or excreted to a very low extent, into oral fluid [108].

In clinical practice, saliva may provide advantages such as: (1) collection is non-invasive, simple and can be performed with a minimum of instruction, (2) sampling can be undertaken at home by patients themselves or by their caregivers and sent to the clinic/hospital in advance of the outpatient clinic visit, (3) it is useful in patients with learning disabilities and is preferred by children and their parents, and (4) it is useful for patients who are afraid of needles or in whom venous access is limited or problematic. Nevertheless, some drawbacks of using oral fluid have been identified: (1) the difficulty in measuring concentrations that may be lower than total serum concentrations, (2) the unacceptability of producing saliva for some patient groups, such as patients on antipsychotic drugs, and (3) the possibility of unreliable results due to the presence of drug residues or blood contamination in the mouth. Therefore, further studies are required to develop an optimal sampling process. Practical considerations should be taken into account including the timing to minimize contamination of oral drug residues or blood contamination, and the method of collection (stimulated or non-stimulated).

\subsubsection{Hair}

While hair analysis has been routinely used for determination of illicit drug use and for forensic cases [109], in the last decade it has been increasingly studied as a biomarker for 
endogenous compounds and medication. The main advantage of hair testing is the prolonged timeframe in which medication use can be retrospectively determined. Moreover, hair concentrations are less sensitive to fluctuation and may therefore be a biomarker of long-term exposure. As scalp hair grows on average $1 \mathrm{~cm}$ per month, the timeframe to be studied is dependent on hair length, and, indeed, its presence on the subject. Factors that can influence drug incorporation may be the patient's age and hair colour, but also drug factors such as it being ionization constant and lipophilicity [110-112]. Further complicating matters are that hair exposure to UV light, chlorine, shampoos and other treatments may lower the hair drug concentration due to drug degradation or extraction [113]. While the sample preparation and result interpretation are considered challenging, it is a unique method that enables access to long-term drug exposure. Indeed, hair has benefits including that it: (1) is easy to collect, (2) requires no invasive technique, (3) does not present a biohazard, and (4) can be stored and shipped at ambient temperature, as the samples are fairly stable and could therefore be collected in remote areas.

This review highlights that hair is predominantly applied for long-term adherence monitoring and mostly applied in HIV/AIDS studies. This finding supports the results of a systematic review that highlighted that hair antiretroviral concentration independently predicts the virologic responses and suggested hair as a biomarker in monitoring antiretroviral medication adherence [114]. Furthermore, hair is also used in psychiatry and epileptic patients. However, in the field of neurological diseases, the dose-response studies that have been performed have mostly varying results, which has been described in more detail in a review on quantitative data [115]. Quantifying the amount of drug intake or daily dose in hair, or adjusting dose levels on it is not a preferable method for TDM because of poor correlations found between administered doses and hair drug concentrations [29, 69]. Yet, this method could still be useful in clinical practice in detection of compliance and drug-taking behaviour.

\subsection{Strengths and Limitations}

In this comprehensive review, we evaluate three emerging bioanalytical methods that have potential to be used in clinical practice. Therefore, all studies were graded on clinical validity on three main points, which we thought sufficient for the intention to provide a high-end overview. This review includes studies from a wide range of publication years (from 2000 to 2020) and all continents, so it captures the growth of assay development and global application of the emerging methods.

Nonetheless, this review also has several limitations. First, there is a possibility that relevant studies were missed, as four new articles were already found with the snowball technique. Articles may have been overlooked that did not state the emerging method in the title, or mention the clinical applicability (TDM and/or MAM) or the use of clinical samples in the abstract. Moreover, only articles that were published in English were included, so additional articles may have been lost in the language selection. Furthermore, the snowballing technique was only applied to a limited number of identified articles, and therefore it is possible that some articles were missed with this method. Second, as this review focuses on drugs measured in real-life situations and real patient populations, we may have missed clinical validations that could be transferable to certain patient groups, for example validations that have been performed in healthy volunteers using medication short term. Third, while the interest for application of these methods is rapidly increasing, the interest for measuring drugs in different matrices is not limited to the last two decades. Much basic analytical research was performed before that and is not included in this review. Additionally, the exclusion of grey literature may have limited the information presented here. However, we found that some of the excluded abstracts were published in full-text form later on and were hence included.

Due to the numerous articles found and the broad coverage, the balance between quality, quantity, and critical appraisal was a challenge. The clinical validation quality labels are a rough estimation of quality and do not take into account some detailed parameters, such as the cross-validation results. Also, no formal bias assessment was performed. Therefore, automated extraction processes would make a broader coverage possible.

\subsection{Future Perspectives}

The findings in this review have implications for the conduct of future research. Most emerging methods described in this review do not yet have clear guidelines for clinical validation, which may limit interpretation of their value, their acceptability and their use. The validation of traditional, established methods (whole blood, serum, plasma) are well described and are used as the golden standard. For DBS, a recent guideline states that the measurement of additional parameters is needed for full validation, especially for routine care measurements [14]. These parameters include number of patients and clinical samples used, quality of the sampling procedure and sample itself, cross-validation, and statistical handling. These guidelines state whether the method can be used interchangeably with the golden standards.

However, comparable guidelines are not yet described for saliva and hair methods. This may be a challenge because the focus of using these methods is not on interchangeability. Cross-validation with measurements of simultaneously 
acquired blood samples is therefore not applicable for all emerging methods, in particular for hair analysis. This review has identified that most of the articles apply a way of cross-validation (if not using blood samples, then mostly by adherence measurements, dose intake, or other clinical outcomes). For saliva, there is no standardisation of stimulation and sample collection, while many different means are available [82]. Some guidelines have, however, been established on sampling quality. For hair, sampling has been described by the society of hair analysis [111], but their focus is more on forensic applications than on MAM or TDM. Before saliva and hair analysis can be successfully applied in routine practice, the general relevant elements for these matrices should also be described to ensure the methods' quality. This review provides useful information for further guideline development, preferably performed by an international society with focus on alternative sampling strategies. Further (systematic) reviews have to be performed to see what methods are currently used and what parameters should be considered for evaluation or even standardisation, and apply these parameters for more detailed study quality assessments.

Furthermore, there are many therapeutic areas such as neurological diseases where DBS and hair can be explored either for MAM or TDM, although a few antipsychotics (i.e., pipamperone and risperidone) have already been shown to be infeasible for TDM using DBS. The use of saliva and hair might also be developed further for respiratory diseases, transplantation, and haemato-oncology particularly for MAM, yet the occurrence of hair loss as an adverse drug reaction might limit the ease of collection in the last two fields.

We recommend:

- All analytical methods should be clinically validated to prove their applicability for routine care

- A clinical validation for emerging methods should include real patient samples acquired from a sufficient number of patients in addition to the performance of a cross-validation with a previously validated method or with valid clinical outcomes.

- Further reviews should consider which other parameters should be used for evaluation and/or standardisation,

- Additional research for the application of emerging methods should be performed for neurological diseases, transplantation and haemato-oncology and respiratory diseases.

- Further research should address the translation from clinical trials to routine patient care.

\section{Conclusions}

Drug concentrations established in DBS, saliva, and hair are increasingly used for MAM and TDM. These matrices differ in their benefits and limitations, yet all have potential for drug monitoring in daily clinical practice. We found several research gaps that provide opportunities for future research. To strengthen comparability and acceptability of these emerging methods, we recommend standardisation of the method of clinical validation.

Supplementary Information The online version contains supplementary material available at https://doi.org/10.1007/s40265-021-01618-7.

\section{Declarations}

Funding The authors received no specific funding for conducting this review. TRZ is supported by a grant from Chiesi Pharmaceutici (Contract No. PA2019-7108) and ZI is supported by the LPDP scholarship (The Indonesian Endowment Fund for Education, Ministry of Finance of Republic of Indonesia, Grant No. 20193220414030), but the funding bodies were not involved in the study design, data collection, analysis, or writing of the manuscript.

Conflict of interest All authors declare that they have no conflicts of interest.

Author contributions TRZ and ZI proposed the outline; performed the literature search, data extraction and data analysis; and wrote the manuscript. CÅ, CTG, SJLB, DJT and JFMB contributed to editing and critically reviewed the manuscript. DJT and JFMB proposed the outline and supervised the project. All authors have proofread and approved the final manuscript.

Ethics approval Not applicable

Informed consent Not applicable

Data availability Data are available upon reasonable request to the corresponding author of the study. The authors intend to set up a more comprehensive database covering all drugs measured in the described emerging methods.

Open Access This article is licensed under a Creative Commons Attribution-NonCommercial 4.0 International License, which permits any non-commercial use, sharing, adaptation, distribution and reproduction in any medium or format, as long as you give appropriate credit to the original author(s) and the source, provide a link to the Creative Commons licence, and indicate if changes were made. The images or other third party material in this article are included in the article's Creative Commons licence, unless indicated otherwise in a credit line to the material. If material is not included in the article's Creative Commons licence and your intended use is not permitted by statutory regulation or exceeds the permitted use, you will need to obtain permission directly from the copyright holder. To view a copy of this licence, visit http://creativecommons.org/licenses/by-nc/4.0/. 


\section{References}

1. World Health Organization. Adherence to long-term therapies: evidence for action [Internet]. 2003 [cited 2021 Jun 28]. https:// www.who.int/chp/knowledge/publications/adherence_report/en/.

2. Osterberg L, Blaschke T. Adherence to medication. N Engl J Med. 2005;353:487-97.

3. Lam WY, Fresco P. Medication adherence measures: an overview. Biomed Res Int. 2015;2015:217047.

4. Stirratt MJ, Curtis JR, Danila MI, Hansen R, Miller MJ, Gakumo CA. Advancing the science and practice of medication adherence. J Gen Intern Med. 2018;33:216-22.

5. Zijp TR, Mol PGM, Touw DJ, van Boven JFM. Smart medication adherence monitoring in clinical drug trials: a prerequisite for personalised medicine? EClinicalMedicine. 2019;15:3-4.

6. Ates HC, Roberts JA, Lipman J, Cass AEG, Urban GA, Dincer C. On-site therapeutic drug monitoring. Trends Biotechnol. 2020;38(11):1262-77.

7. Kang JS, Lee MH. Overview of therapeutic drug monitoring. Korean J Intern Med. 2009;24(1):1-10.

8. Galena HJ. Complications occurring from diagnostic venipuncture. J Fam Pract. 1992;34(5):582-4.

9. Esteve-Romero J, Albiol-Chiva J, Peris-Vicente J. A review on development of analytical methods to determine monitorable drugs in serum and urine by micellar liquid chromatography using direct injection. Anal Chim Acta. 2016;926:1-16.

10. Argoff CE, Alford DP, Fudin J, Adler JA, Bair MJ, Dart RC, et al. Rational urine drug monitoring in patients receiving opioids for chronic pain: consensus recommendations. Pain Med. 2018;19(1):97-117.

11. Cohen AN, Collins G, Nucifora FC, Strobel R, Wait DB, Young AS. Clinical consensus recommendations for urine testing of adherence to antipsychotics among people with serious mental illness. Psychiatr Serv. 2018;69(3):345-8.

12. U.S. Department of Health and Human Services, Food and Drug Administration. Bioanalytical method validation guidance for industry. U.S. Department of Health and Human Services and Food and Drug Administration. 2018. p. 1-41.

13. European Medicines Agency. Guideline on bioanalytical method validation. 2011;44:1-23.

14. Capiau S, Veenhof H, Koster RA, Bergqvist Y, Boettcher M, Halmingh $\mathrm{O}$, et al. Official International Association for Therapeutic Drug Monitoring and Clinical Toxicology guideline: development and validation of dried blood spot-based methods for therapeutic drug monitoring. Ther Drug Monit. 2019;41(4):409-30.

15. Vindrola-Padros C, Sidhu MS, Georghiou T, Sherlaw-Johnson C, Singh KE, Tomini SM, et al. The implementation of remote home monitoring models during the COVID-19 pandemic in England. EClinicalMedicine. 2021;34:100799.

16. Arksey H, O'Malley L. Scoping studies: towards a methodological framework. Int J Soc Res Methodol Theory Pract. 2005;8(1):19-32.

17. Tricco AC, Lillie E, Zarin W, O'Brien KK, Colquhoun H, Levac $\mathrm{D}$, et al. PRISMA extension for scoping reviews (PRISMA-ScR): checklist and explanation. Ann Intern Med. 2018;169(7):467-73.

18. Ouzzani M, Hammady H, Fedorowicz Z, Elmagarmid A. Rayyan — a web and mobile app for systematic reviews. Syst Rev. 2016;5:210.

19. Geers LM, Cohen D, Wehkamp LM, Van Hateren K, Koster RA, Fedorenko OY, et al. Dried blood spot analysis for therapeutic drug monitoring of clozapine. J Clin Psychiatry. 2017;78(9):e1211-8.

20. da Silva ACC, Raasch JR, Vargas TG, Peteffi GP, Hahn RZ, Antunes MV, et al. Simultaneous determination of fluoxetine and norfluoxetine in dried blood spots using high-performance liquid chromatography-tandem mass spectrometry. Clin Biochem. 2018;52:85-93.

21. Courlet P, Decosterd LA, Brown JA, Saldanha SA, Marzolini $\mathrm{C}$, Cavassini M, et al. Emtricitabine and lamivudine concentrations in saliva: a simple suitable test for treatment adherence. $\mathrm{J}$ Antimicrob Chemother. 2019;74(8):2468-70.

22. Böttcher M, Kühne D, Beck O. Compliance testing of patients in ADHD treatment with lisdexamphetamine (Elvanse ${ }^{\circledR}$ ) using oral fluid as specimen. Clin Mass Spectrom. 2019;14:99-105.

23. Idkaidek N, Hamadi S, El-Assi M, Al-Shalalfeh A, AlGhazawi A. Saliva versus plasma therapeutic drug monitoring of pregabalin in Jordanian patients. Drug Res (Stuttg). 2018;68(10):596-600.

24. Ghimire S, Maharjan B, Jongedijk EM, Kosterink JGW, Ghimire GR, Touw DJ, et al. Evaluation of saliva as a potential alternative sampling matrix for therapeutic drug monitoring of levofloxacin in patients with multidrug-resistant tuberculosis. Antimicrob Agents Chemother. 2019;63(5):e02379-e2418.

25. Ransohoff JR, Petrides AK, Piscitello GJ, Flood JG, Melanson SEF. Urine is superior to oral fluid for detecting buprenorphine compliance in patients undergoing treatment for opioid addiction. Drug Alcohol Depend. 2019;203:8-12.

26. Boons CCLM, Chahbouni A, Schimmel AM, Wilhelm AJ, den Hartog YM, Janssen JJWM, et al. Dried blood spot sampling of nilotinib in patients with chronic myeloid leukaemia: a comparison with venous blood sampling. J Pharm Pharmacol. 2017;69(10):1265-74.

27. Veenhof H, Koster RA, Alffenaar JWC, Berger SP, Bakker SJL, Touw DJ. Clinical validation of simultaneous analysis of tacrolimus, cyclosporine A, and creatinine in dried blood spots in kidney transplant patients. Transplantation. 2017;101(7):1727-33.

28. Duthaler U, Berger B, Erb S, Battegay M, Letang E, Gaugler $\mathrm{S}$, et al. Using dried blood spots to facilitate therapeutic drug monitoring of antiretroviral drugs in resource-poor regions. J Antimicrob Chemother. 2018;73(10):2729-37.

29. Braal CL, Veerman GDM, Peric R, Aerts JGJV, Mathijssen RHJ, Koolen SLW, et al. Quantification of the tyrosine kinase inhibitor erlotinib in human scalp hair by liquid chromatography-tandem mass spectrometry: Pitfalls for clinical application. J Pharm Biomed Anal. 2019;172:175-82.

30. Sun X, Wang L, Yang F, Ren J, Jiang P, Liu H, et al. Correlation of hair risperidone concentration and serum level among patients with schizophrenia. Gen Psychiatry. 2019;32(1):e100042.

31. Hassall D, Brealey N, Wright W, Hughes S, West A, Ravindranath $\mathrm{R}$, et al. Hair analysis to monitor adherence to prescribed chronic inhaler drug therapy in patients with asthma or COPD. Pulm Pharmacol Ther. 2018;51:59-64.

32. Mave V, Kinikar A, Kagal A, Nimkar S, Koli H, Khwaja S, et al. Isoniazid concentrations in hair and plasma area-under-thecurve exposure among children with tuberculosis. PLoS ONE. 2017;12(12):e0189101.

33. Chawana TD, Gandhi M, Nathoo K, Ngara B, Louie A, Horng $\mathrm{H}$, et al. Defining a cutoff for atazanavir in hair samples associated with virological failure among adolescents failing secondline antiretroviral treatment. J Acquir Immune Defic Syndr. 2017;76(1):55-9.

34. Harris PA, Taylor R, Thielke R, Payne J, Gonzalez N, Conde JG. Research electronic data capture (REDCap)-A metadatadriven methodology and workflow process for providing translational research informatics support. J Biomed Inform. 2009;42(2):377-81.

35. Harris PA, Taylor R, Minor BL, Elliott V, Fernandez M, O'Neal $\mathrm{L}$, et al. The REDCap consortium: building an international community of software platform partners. J Biomed Inform. 2019;95:103208. 
36. Foerster KI, Huppertz A, Meid AD, Müller OJ, Rizos T, Tilemann L, et al. Dried-blood-spot technique to monitor direct oral anticoagulants: clinical validation of a UPLC-MS/MS-based assay. Anal Chem. 2018;90(15):9395-402.

37. van den Elsen SHJ, Akkerman OW, Jongedijk EM, Wessels M, Ghimire S, van der Werf TS, et al. Therapeutic drug monitoring using saliva as matrix: an opportunity for linezolid, but challenge for moxifloxacin. Eur Respir J. 2020;55(5):1901903.

38. Kromdijk W, Mulder JW, Smit PM, Ter Heine R, Beijnen JH, Huitema AD, et al. Therapeutic drug monitoring of antiretroviral drugs at home using dried blood spots: a proof-of-concept study. Antivir Ther. 2013;18(6):821-5.

39. Al-Uzri A, Freeman KA, Wade J, Clark K, Bleyle LA, Munar $\mathrm{M}$, et al. Longitudinal study on the use of dried blood spots for home monitoring in children after kidney transplantation. Pediatr Transplant. 2017;21(6):e12983.

40. Zwart TC, Gokoel SRM, van der Boog PJM, de Fijter JW, Kweekel DM, Swen JJ, et al. Therapeutic drug monitoring of tacrolimus and mycophenolic acid in outpatient renal transplant recipients using a volumetric dried blood spot sampling device. Br J Clin Pharmacol. 2018;84(12):2889-902.

41. Hawwa AF, AlBawab AQ, Rooney M, Wedderburn LR, Beresford MW, McElnay JC. A novel dried blood spot-LCMS method for the quantification of methotrexate polyglutamates as a potential marker for methotrexate use in children. PLoS ONE. 2014;9(2):e89908.

42. Angelakis E, Armstrong N, Nappez C, Richez M, Chabriere E, Raoult D. Doxycycline assay hair samples for testing long-term compliance treatment. J Infect. 2015;71(5):511-7.

43. Gandhi M, Glidden DV, Liu A, Anderson PL, Horng H, Defechereux P, et al. Strong correlation between concentrations of tenofovir (TFV) emtricitabine (FTC) in hair and TFV diphosphate and FTC triphosphate in dried blood spots in the iPrEx open label extension: Implications for pre-exposure prophylaxis adherence monitoring. J Infect Dis. 2015;212(9):1402-6.

44. Karaś-Ruszczyk K, Kuczyńska J, Sienkiewicz-Jarosz H, Kurkowska-Jastrzębska I, Bienkowski P, Restel M, et al. Comparison of plasma, saliva, and hair levetiracetam concentrations. Ther Drug Monit. 2017;39(3):263-8.

45. Kuczynska J, Karas-Ruszczyk K, Zakrzewska A, Dermanowski M, Sienkiewicz-Jarosz H, Kurkowska-Jastrzebska I, et al. Comparison of plasma, saliva, and hair lamotrigine concentrations. Clin Biochem. 2019;74:24-30.

46. Fucci N, De Giovanni N. Methadone in hair and sweat from patients in long-term maintenance therapy. Ther Drug Monit. 2007;29(4):452-4.

47. Cirimele V, Kintz P, Gosselin O, Ludes B. Clozapine dose-concentration relationships in plasma, hair and sweat specimens of schizophrenic patients. Forensic Sci Int. 2000;107(1-3):289-300.

48. Henkin RI. Comparative monitoring of oral theophylline treatment in blood serum, saliva, and nasal mucus. Ther Drug Monit. 2012;34(2):217-21.

49. Veenhof H, Koster RA, Junier LAT, Berger SP, Bakker SJL, Touw DJ. Volumetric absorptive microsampling and dried blood spot microsampling vs. conventional venous sampling for tacrolimus trough concentration monitoring. Clin Chem Lab Med. 2020;58(10):1687-95.

50. Guo M-Z, Shao L, Xi C, Li H-J, Wang L, Pan Y-J, et al. Assay of dried blood spot from finger prick for sodium valproate via ink auxiliary headspace gas chromatography mass spectrometry. J Chromatogr A. 2019;1601:335-9.

51. Alcaide ML, Ramlagan S, Rodriguez VJ, Cook R, Peltzer K, Weiss SM, et al. Self-report and dry blood spot measurement of antiretroviral medications as markers of adherence in pregnant women in rural South Africa. AIDS Behav. 2017;21(7):2135-40.
52. Shah NM, Hawwa AF, Millership JS, Collier PS, Ho P, Tan ML, et al. Adherence to antiepileptic medicines in children: A multiple-methods assessment involving dried blood spot sampling. Epilepsia. 2013;54(6):1020-7.

53. van Boekel GAJ, Donders ART, Hoogtanders KEJ, Havenith TRA, Hilbrands LB, Aarnoutse RE. Limited sampling strategy for prolonged-release tacrolimus in renal transplant patients by use of the dried blood spot technique. Eur J Clin Pharmacol. 2015;71(7):811-6.

54. Zhang Q, Li X, Qiao S, Shen Z, Zhou Y. Comparing self-reported medication adherence measures with hair antiretroviral concentration among people living with HIV in Guangxi, China. AIDS Res Ther. 2020;17(1):8.

55. Capron A, Antunes MV, Wagner SC, Mattevi VS, Vieira N, Leite $\mathrm{R}$, et al. First report of imatinib measurement in hair: Method development and preliminary evaluation of the relation between hair and plasma concentrations with therapeutic response in chronic myeloid leukemia. Clin Chim Acta. 2016;453:42-7.

56. Ferrari A, Baraldi C, Licata M, Vandelli D, Marchesi F, Palazzoli F, et al. Hair analysis for detection of triptans occasionally used or overused by migraine patients-a pilot study. Eur J Clin Pharmacol. 2016;72(9):1075-81.

57. Tsatsakis AM, Psillakis T, Paritsis N. Phenytoin concentration in head hair sections: a method to evaluate the history of drug use. J Clin Psychopharmacol. 2000;20(5):560-73.

58. Peeters LEJ, Feyz L, Hameli E, Zwart T, Bahmany S, Daemen $\mathrm{J}$, et al. Clinical validation of a dried blood spot assay for 8 antihypertensive drugs and 4 active metabolites. Ther Drug Monit. 2019;42(3):460-7.

59. Shaparin N, Mehta N, Kunkel F, Stripp R, Borg D, Kolb E. A novel chronic opioid monitoring tool to assess prescription drug steady state levels in oral fluid. Pain Med. 2017;18(11):2162-9.

60. Neumann J, Beck O, Dahmen N, Boettcher M. Potential of oral fluid as a clinical specimen for compliance monitoring of psychopharmacotherapy. Ther Drug Monit. 2018;40(2):245-51.

61. Gandhi M, Ameli N, Bacchetti P, Gange SJ, Anastos K, Levine $\mathrm{A}$, et al. Protease inhibitor levels in hair strongly predict virologie response to treatment. AIDS. 2009;23(4):471-8.

62. Pintye J, Bacchetti P, Teeraananchai S, Kerr S, Prasitsuebsai W, Singtoroj T, et al. Brief report: lopinavir hair concentrations are the strongest predictor of viremia in HIV-infected Asian children and adolescents on second-line antiretroviral therapy. J Acquir Immune Defic Syndr. 2017;76(4):367-71.

63. van Zyl GU, van Mens TE, McIlleron H, Zeier M, Nachega JB, Decloedt E, et al. Low lopinavir plasma or hair concentrations explain second-line protease inhibitor failures in a resource-limited setting. J Acquir Immune Defic Syndr. 2011;56(4):333-9.

64. Yang H, Chu L, Wu Y, Wang W, Yang J, Zhang Q, et al. LC-MS/ MS quantification of nevirapine and its metabolites in hair for assessing long-term adherence. Molecules. 2020;25(23):5692.

65. Gandhi M, Murnane PM, Bacchetti P, Elion R, Kolber MA, Cohen SE, et al. Hair levels of preexposure prophylaxis drugs measure adherence and are associated with renal decline among men/transwomen. AIDS. 2017;31(16):2245-51.

66. Kloosterboer SM, De Winter BCM, Bahmany S, Al-Hassany L, Dekker A, Dieleman GC, et al. Dried blood spot analysis for therapeutic drug monitoring of antipsychotics: drawbacks of its clinical application. Ther Drug Monit. 2018;40(3):344-50.

67. Fisher DS, Beyer C, Van Schalkwyk G, Seedat S, Flanagan RJ. Measurement of clozapine, norclozapine, and amisulpride in plasma and in oral fluid obtained using 2 different sampling systems. Ther Drug Monit. 2017;39(2):109-17.

68. Patteet L, Maudens KE, Morrens M, Sabbe B, Dom G, Neels $\mathrm{H}$. Determination of common antipsychotics in quantisal-collected oral fluid by UHPLC-MS/MS: Method validation and 
applicability for therapeutic drug monitoring. Ther Drug Monit. 2016;38(1):87-97.

69. Müller A, Jungen H, Iwersen-Bergmann S, Sterneck M, Andresen-Streichert H, et al. Analysis of cyclosporin A in hair samples from liver transplanted patients. Ther Drug Monit. 2013;35(4):450-8.

70. Wohkittel C, Högger P, Fekete S, Romanos M, Gerlach M. Relationship between amphetamine concentrations in saliva and serum in children and adolescents with attention-deficit/hyperactivity disorder. Ther Drug Monit. 2021;43(4)564-69.

71. Hardy J, Norris R, Anderson H, O'Shea A, Charles B. Is saliva a valid substitute for plasma in pharmacokinetic studies of oxycodone and its metabolites in patients with cancer? Support Care Cancer. 2012;20(4):767-72.

72. Wang X, Zhuo Y, Tang X, Qiang H, Liu W, Wu H, et al. Segmental analysis of antidepressant and antipsychotic drugs in the hair of schizophrenic patients. Drug Test Anal. 2019;12(4):472-84.

73. Hugen PWH, Burger DM, de Graaff M, ter Hofstede HJM, Hoetelmans RMW, Brinkman K, et al. Saliva as a specimen for monitoring compliance but not for predicting plasma concentrations in patients with HIV treated with indinavir. Ther Drug Monit. 2000;22(4):437-45.

74. Stegmann B, Dörfelt A, Haen E. Quantification of methylphenidate, dexamphetamine, and atomoxetine in human serum and oral fluid by HPLC with fluorescence detection. Ther Drug Monit. 2016;38(1):98-107.

75. Brooks E, Tett SE, Isbel NM, McWhinney B, Staatz CE. Investigation of the association between total and free plasma and saliva mycophenolic acid concentrations following administration of enteric-coated mycophenolate sodium in adult kidney transplant recipients. Clin Drug Investig. 2019;39(12):1175-84.

76. de Truchis P, Le MP, Daou M, Madougou B, Nouhou Y, Saley SM, et al. High efficacy of first-line ART in a West African cohort, assessed by dried blood spot virological and pharmacological measurements. J Antimicrob Chemother. 2016;71(11):3222-7.

77. Pappadopulos E, Jensen PS, Chait AR, Arnold LE, Swanson JM, Greenhill LL, et al. Medication adherence in the MTA: Saliva methylphenidate samples versus parent report and mediating effect of concomitant behavioral treatment. J Am Acad Child Adolesc Psychiatry. 2009;48(5):501-10.

78. Gandhi M, Devi S, Bacchetti P, Chandy S, Heylen E, Phung $\mathrm{N}$, et al. Measuring adherence to antiretroviral therapy via hair concentrations in India. J Acquir Immune Defic Syndr. 2019;81(2):202-6.

79. Gandhi M, Greenblatt RM, Bacchetti P, Jin C, Huang Y, Anastos $\mathrm{K}$, et al. A single-nucleotide polymorphism in CYP2B6 leads to $>3$-fold increases in efavirenz concentrations in plasma and hair among HIV-infected women. J Infect Dis. 2012;206(9):1453-61.

80. Prasitsuebsai W, Kerr SJ, Truong KH, Ananworanich J, Do VC, Van NL, et al. Using lopinavir concentrations in hair samples to assess treatment outcomes on second-line regimens among Asian children. AIDS Res Hum Retrovirus. 2015;31(10):1009-14.

81. Hugen PWH, Burger DM, Aarnoutse RE, Baede PA, Nieuwkerk PT, Koopmans PP, et al. Therapeutic drug monitoring of HIVprotease inhibitors to assess noncompliance. Ther Drug Monit. 2002;24(5):579-87.

82. Bellagambi FG, Lomonaco T, Salvo P, Vivaldi F, Hangouët M, Ghimenti S, et al. Saliva sampling: methods and devices. An overview. Trends Anal Chem. 2020;124:115781.

83. Veringa A, ter Avest M, Touw DJ, Alffenaar JWC. Comment on: utility of voriconazole therapeutic drug monitoring: a metaanalysis. J Antimicrob Chemother. 2016;71(11):3316-7.

84. ter Heine R, Beijnen JH, Huitema ADR. Bioanalytical issues in patient-friendly sampling methods for therapeutic drug monitoring: focus on antiretroviral drugs. Bioanalysis. 2009;1(7):1329-38.

85. Duthaler U, Berger B, Erb S, Battegay M, Letang E, Gaugler S, et al. Automated high throughput analysis of antiretroviral drugs in dried blood spots. J Mass Spectrom. 2017;52(8):534-42.

86. Hoogtanders K, van der Heijden J, Christiaans M, van de Plas A, van Hooff J, Stolk L. Dried blood spot measurement of tacrolimus is promising for patient monitoring. Transplantation. 2007;83(2):237-8.

87. Wilhelm AJ, Klijn A, Den Burger JCG, Visser OJ, Veldkamp AI, Janssen JJWM, et al. Clinical validation of dried blood spot sampling in therapeutic drug monitoring of ciclosporin a in allogeneic stem cell transplant recipients: direct comparison between capillary and venous sampling. Ther Drug Monit. 2013;35(1):92-5.

88. Arpini J, Antunes MV, Pacheco LS, Gnatta D, Rodrigues MF, Keitel E, et al. Clinical evaluation of a dried blood spot method for determination of mycophenolic acid in renal transplant patients. Clin Biochem. 2013;46(18):1905-8.

89. Willemsen AECAB, Knapen LM, de Beer YM, Bruggemann RJM, Croes S, van Herpen CML, et al. Clinical validation study of dried blood spot for determining everolimus concentration in patients with cancer. Eur J Clin Pharmacol. 2018;74(4):465-71.

90. Antunes M, Raymundo S, Wagner S, Mattevi VS, Vieira N, Leite $\mathrm{R}$, et al. DBS sampling in imatinib therapeutic drug monitoring: from method development to clinical application. Bioanalysis. 2015;7(16):2105-17.

91. de Wit D, den Hartigh J, Gelderblom H, Qian Y, den Hollander M, Verheul H, et al. Dried blood spot analysis for therapeutic drug monitoring of pazopanib. J Clin Pharmacol. 2015;55(12):1344-50.

92. Raymundo S, Muller VV, Andriguetti NB, Tegner M, Artmann AC, Kluck HM, et al. Determination of docetaxel in dried blood spots by LC-MS/MS: method development, validation and clinical application. J Pharm Biomed Anal. 2018;157:84-91.

93. Martial LC, Hoogtanders KEJ, Schreuder MF, Cornelissen EA, Van Der Heijden J, Joore MA, et al. Dried blood spot sampling for tacrolimus and mycophenolic acid in children: analytical and clinical validation. Ther Drug Monit. 2017;39(4):412-21.

94. Dickerson JA, Sinkey M, Jacot K, Stack J, Sadilkova K, Law YM, et al. Tacrolimus and sirolimus in capillary dried blood spots allows for remote monitoring. Pediatr Transplant. 2015;19(1):101-6.

95. Koster RA, Veenhof H, Botma R, Hoekstra AT, Berger SP, Bakker SJ, et al. Dried blood spot validation of five immunosuppressants, without hematocrit correction, on two LC-MS/MS systems. Bioanalysis. 2017;9(7):553-63.

96. Kromdijk W, Mulder JW, Rosing H, Smit PM, Beijnen JH, Huitema ADRR. Use of dried blood spots for the determination of plasma concentrations of nevirapine and efavirenz. J Antimicrob Chemother. 2012;67(5):1211-6.

97. Saracino MA, Marcheselli C, Somaini L, Pieri MC, Gerra G, Ferranti A, et al. A novel test using dried blood spots for the chromatographic assay of methadone. Anal Bioanal Chem. 2012;404(2):503-11.

98. Tey HY, See HH. A review of recent advances in microsampling techniques of biological fluids for therapeutic drug monitoring. J Chromatogr A. 2021;1635:461731.

99. Denniff P, Spooner N. Volumetric absorptive microsampling: a dried sample collection technique for quantitative bioanalysis. Anal Chem. 2014;86(16):8489-95.

100. Nakahara T, Otani N, Ueno T, Hashimoto K. Development of a hematocrit-insensitive device to collect accurate volumes of dried blood spots without specialized skills for measuring clozapine and its metabolites as model analytes. J Chromatogr B Anal Technol Biomed Life Sci. 2018;1087-1088:70-9. 
101. Caporossi L, Santoro A, Papaleo B. Saliva as an analytical matrix: State of the art and application for biomonitoring. Biomarkers. 2010;15(6):475-87.

102. van Heeswijk RP, Veldkamp AI, Mulder JW, Meenhorst PL, Beijnen JH, Lange JM, et al. Saliva as an alternative body fluid for therapeutic drug monitoring of the nonnucleoside reverse transcription inhibitor nevirapine. Ther Drug Monit. 2001;23(3):255-8.

103. Kiang TKL, Ensom MHH. A qualitative review on the pharmacokinetics of antibiotics in saliva: Implications on clinical pharmacokinetic monitoring in humans. Clin Pharmacokinet. 2016;55(3):313-58.

104. Mendonza AE, Gohh RY, Akhlaghi F. Analysis of mycophenolic acid in saliva using liquid chromatography tandem mass spectrometry. Ther Drug Monit. 2006;28(3):402-6.

105. O'Neal CL, Crouch DJ, Rollins DE, Fatah AA. The effects of collection methods on oral fluid codeine concentrations. J Anal Toxicol. 2000;24:536-42.

106. Ghareeb M, Gohh RY, Akhlaghi F. Tacrolimus concentration in saliva of kidney transplant recipients: Factors influencing the relationship with whole blood concentrations. Clin Pharmacokinet. 2018;57(9):1199-210.

107. Yamada E, Takagi R, Tanabe Y, Fujiwara H, Hasegawa N, Kato S. Plasma and saliva concentrations of abacavir, tenofovir, darunavir, and raltegravir in HIV-1-infected patients. Int J Clin Pharmacol Ther. 2017;55(7):567-70.

108. Richter LHJ, Jacobs CM, Mahfoud F, Kindermann I, Böhm M, Meyer MR. Development and application of a LC-HRMS/MS method for analyzing antihypertensive drugs in oral fluid for monitoring drug adherence. Anal Chim Acta. 2019;1070:69-79.

109. Kintz P. Hair analysis in forensic toxicology. Wiley Interdiscip Rev Forensic Sci. 2018;1(1):e1196.

110. Nakahara Y, Kikura R. Hair analysis for drugs of abuse. XIII. Effect of structural factors on incorporation of drugs into hair: the incorporation rates of amphetamine analogs. Arch Toxicol. 1996;70(12):841-9.

111. Cooper GAA, Kronstrand R, Kintz P. Society of Hair Testing guidelines for drug testing in hair. Forensic Sci Int. 2012;218(1-3):20-4.

112. Shima N, Nitta A, Kamata T, Sasaki K, Matsuta S, Ishikawa $A$, et al. Incorporation of zolpidem and methoxyphenamine into white hair strands after single administrations: influence of hair pigmentation on drug incorporation. Forensic Sci Int. 2019;301:67-75.

113. Jurado C, Kintz P, Menéndez M, Repetto M. Influence of the cosmetic treatment of hair on drug testing. Int J Legal Med. 1997;110:159-63.

114. Zhang Q, Qiao S, Yang X, Li X. Antiretroviral concentration in hair as a measure for antiretroviral medication adherence: a systematic review of global literature. AIDS Behav. 2019;24(1):311-30.

115. Methling M, Krumbiegel F, Hartwig S. Hair analysis of antidepressants and antipsychotics-Overview of quantitative data. Drug Test Anal. 2020;12(6):659-76.

\section{Authors and Affiliations}

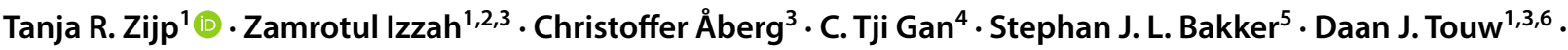 Job F. M. van Boven ${ }^{1,6}$}

1 University of Groningen, University Medical Center Groningen, Department of Clinical Pharmacy and Pharmacology, Groningen, The Netherlands

2 Department of Pharmacy Practice, Faculty of Pharmacy, Universitas Airlangga, Surabaya, Indonesia

3 University of Groningen, Groningen Research Institute of Pharmacy, Department of Pharmaceutical Analysis, Groningen, The Netherlands
4 University of Groningen, University Medical Center Groningen, Respiratory Diseases and Lung Transplantation, Groningen, The Netherlands

5 University of Groningen, University Medical Center Groningen, Department of Internal Medicine, Division of Nephrology, Groningen, The Netherlands

6 Medication Adherence Expertise Center of the Northern Netherlands (MAECON), Groningen, The Netherlands 